\title{
A Collisional Radiative model for low-pressure hydrogen-caesium plasmas and its application to an RF source for negative hydrogen ions
}

\author{
D. Wünderlich ${ }^{\mathrm{a}, *}$, C. Wimmer ${ }^{\mathrm{a}}$, R. Friedl ${ }^{\mathrm{b}}$ \\ ${ }^{a}$ Max-Planck-Institut für Plasmaphysik (IPP), Boltzmannstr. 2, D-85748 Garching, \\ Germany \\ ${ }^{b} A G$ Experimentelle Plasmaphysik, Universität Augsburg, Universitätsstr. 1, D-86135 \\ Augsburg, Germany
}

\begin{abstract}
A collisional radiative $(\mathrm{CR})$ model for caesium in low-temperature, lowpressure hydrogen-caesium plasmas is introduced. This model includes the caesium ground state, 14 excited states, the singly charged caesium ion and the negative hydrogen ion. The reaction probabilities needed as input are based on literature data, using some scaling and extrapolations. Additionally, new cross sections for electron collision ionization and three-body recombination have been calculated.

The relevance of mutual neutralization of positive caesium ions and negative hydrogen ions is highlighted: depending on the densities of the involved particle species, this excitation channel can have a significant influence on the population densities of excited states in the caesium atom. This strong influence is successfully verified by optical emission spectroscopy measurements performed at the IPP prototype source for negative hydrogen ions.

As a consequence, population models for caesium in electronegative lowtemperature, low-pressure hydrogen-caesium plasmas need to take into account the mutual neutralization process. The present CR model is an example for such models and represents an important prerequisite for deducing the total caesium density in such plasmas.
\end{abstract}

Keywords: Hydrogen-caesium plasma, Low temperature plasma, Low

\footnotetext{
*Corresponding author. Tel.: +49 893299 1916; fax.: +49 893299961916

Email address: dirk.wuenderlich@ipp.mpg.de (D. Wünderlich)
} 
pressure plasma, Collisional radiative model, Mutual neutralization, Optical emission spectroscopy

\section{Introduction}

Caesium is the most electropositive non-radioactive alkali metal. Its work function is low $(2.14 \mathrm{eV}$ for bulk polycrystalline caesium[1]) and thus it is a very effective electron donor. This property is widely used for a variety of applications: pure caesium or caesium compounds like caesium iodide or caesium oxide are used in photoelectric devices like photomultipliers or photodiodes in order to increase the quantum efficiency and thus the sensitivity of the detector[2, 3].

Another field of application is the generation of negative ions by charge exchange of positive ions or atoms with caesium. Such charge exchange processes can either happen in a caesium gas target[4] or on a converter surface covered by a caesium layer[5]. The latter reaction - surface production of negative ions - is applied in particle sources for accelerators $[6,7]$ and in sources for negative hydrogen or deuterium ions used for the neutral beam injection (NBI) system in fusion experiments as LHD[8], JT60-U[9] or the future fusion device ITER $[10,11,12]$. In such surface production based sources for negative hydrogen ions the caesiated converter surface is in contact with a low-temperature, low-pressure hydrogen-caesium plasma: typically the electron temperature is below a few eV and the gas pressure is well below $1 \mathrm{~Pa}$. Caesium is a minority species in the hydrogen background and thus the partial pressure of caesium is significantly smaller.

The efficiency for the surface production process depends inversely exponential on the surface work function[13]. Thus, desirable for obtaining a high negative ion yield is a work function as low as possible at the convertor surface. The work function of caesiated surfaces depends on the substrate material, the thickness of the caesium layer and the amount of impurities (from the plasma or the background gas) embedded into the layer. For pure caesium layers on metal surfaces a minimum work function is obtained for a layer thickness of well below one monolayer (ML)[14]. For example, 0.6 ML of caesium on a W(100) surface (bulk work function of tungsten: $4.52 \mathrm{eV}[14]$ ) result in a work function of $1.6 \mathrm{eV}$ - which is significantly lower than the work function for bulk caesium. For thick pure caesium layers (above one ML) the work function approaches the work function of bulk caesium. Embedded impurities can result either in a decreased or increased work function compared 
to clean layers: while the work function for $\mathrm{Cs}_{11} \mathrm{O}_{3}$ is $1.35 \mathrm{eV}[15]$, a work function of $2.2 \mathrm{eV}$, i.e. a value slightly above the one for bulk caesium, was observed[16] in a plasma environment similar to the one in negative hydrogen ion sources for NBI.

The caesium needed for obtaining the low work function is introduced to the ion sources by a caesium injection system containing a liquid[17] or solid[18] caesium reservoir. It is deposited at the inner surfaces of the source from which it is redistributed mainly by the influence of the plasma[19]. This redistribution process is strongly affected by the wall surface temperatures and the caesium chemistry, i.e. the formation of caesium compounds and the inclusion of impurities into the caesium layers at the surfaces[20].

Achieving and sustaining a low work function of the caesium-covered converter surface is one key issue in operating negative ion sources for fusion experiments. Thus, highly desirable is a diagnostic technique for determining in-situ the work function of the converter. Although several techniques exist[21] for measuring the work function of a surface, these cannot be applied in a plasma environment due to interference of the plasma with the diagnostic or vice versa[16]. Due to different reasons (e.g. interference of the measurement device with the particle fluxes responsible for the caesium redistribution) pulsed operation of plasma and work function diagnostics as described in [16] for a small laboratory experiment - has up to now never been applied in negative ion sources for fusion.

One important factor for reducing the work function (and maintaining this reduced work function) is a sufficient influx of fresh caesium onto the converter[16]. This caesium flux is correlated to the caesium density in the plasma volume. Thus, monitoring the caesium density in the plasma close to the converter surface is a promising technique for indirectly characterizing the converter work function. A diagnostic technique for the caesium density in a plasma is optical emission spectroscopy (OES)[22]. Depending on the intensity of the emitted light and the sensitivity of the detector, the population densities of some of the electronically excited states in the atom and the singly charged ion are accessible.

In order to deduce the total density of caesium (including the excited states and the ions), a population model has to be applied. The most simple kind of population models are corona models - balancing electron collision excitation from the ground state with spontaneous emission. More sophisticated are collisional radiative (CR) models where all exciting and de-exciting processes relevant (i.e. with a non-negligible reaction rate) in the plasma un- 
der investigation are balanced.

Some CR models for caesium have been described in the literature[23, $24,25]$. These models are based on reaction cross sections calculated using rather simplistic techniques, implying a large error bar of the cross sections itself (mainly close to the threshold energy) and consequently also of the CR model results. Additionally, the processes implemented to these models are appropriate only for describing pure caesium plasmas and processes relevant for hydrogen-caesium plasmas are not taken into account. One important example is mutual neutralization of positive caesium ions with negative hydrogen ions[26] - ending up in an excited caesium atom and a hydrogen atom in the ground state.

This paper describes a caesium CR model based on the flexible solver Yacora[27] and a new and comprehensive set of input data. This model predicts a high relevance of mutual neutralization for the population densities of excited states of caesium in the low-temperature, low-pressure hydrogencaesium plasmas of negative hydrogen ion sources. In a second step, this predicted high relevance of mutual neutralization is experimentally demonstrated by applying the CR model to results of OES measurements at the IPP negative ion source prototype[12, 28] for ITER NBI.

\section{The CR model for caesium}

\subsection{Physics of the CR model for caesium}

CR models describe how the population densities of excited states in atoms or molecules depend on plasma parameters like the electron temperature $T_{\mathrm{e}}$, the electron density $n_{\mathrm{e}}$, the ground state density and the densities of all other particles involved in populating and depopulating the excited states[29, 30, 31]. One important area of application for CR models is the interpretation of diagnostic results: by comparing measured population densities (determined e.g. by OES) with model results the plasma parameters can be deduced [22, 27].

In general, CR models include a system of coupled ordinary differential equations describing the temporal evolution of the population densities of all excited states included to the model. These so-called rate equations balance the reaction rates of processes responsible for exciting and de-exciting excited states in the atom or molecule. Included to the model should be all excited states for that the population densities are to be calculated, e.g. the upper states of transitions accessible by OES. Which other excited states 


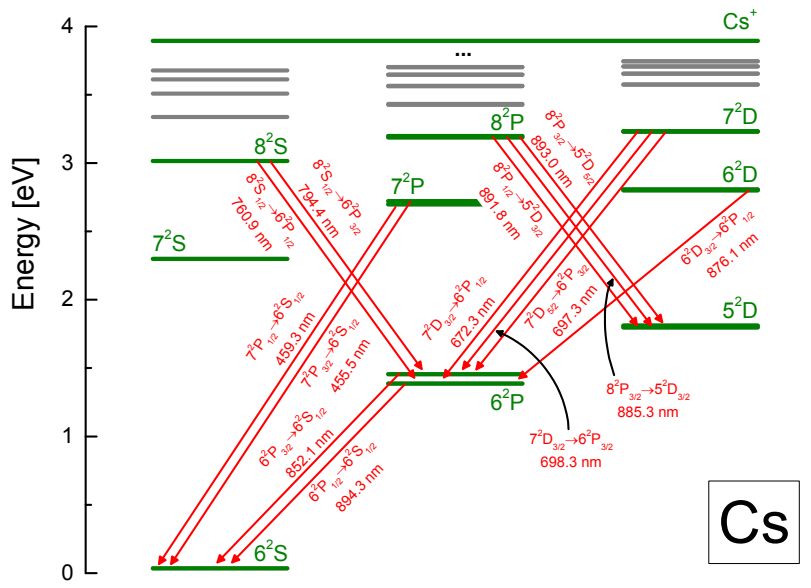

Figure 1: Energy level diagram of the caesium atom. Indicated (in green) are the states implemented to the CR model and all optically allowed transitions between $\lambda=400 \mathrm{~nm}$ and $\lambda=900 \mathrm{~nm}$ and with $A_{i k}>10^{4} \mathrm{~s}^{-1}$ originating from these states.

are included to the model usually is the result of a trade-off between precise description of processes relevant for exciting and de-exciting the states of interest and the availability and accuracy of the reaction probabilities needed for formulating the equations.

Shown in figure 1 is an energy level diagram of caesium. The following 16 states included to the CR model for caesium in low-temperature, lowpressure hydrogen-caesium plasmas are indicated in green: the ground state $6^{2} \mathrm{~S}$, all electronically excited states with an excitation energy $E_{\text {ex }}<3.3 \mathrm{eV}$ and orbital angular momentum quantum number $L \leq 2$ (i.e. up to the $\mathrm{D}$ states) and the singly charged ion $\mathrm{Cs}^{+}$. Table 1 summarizes these states and their excitation energies[32].

Reason for taking into account also the ion is that the first ionization threshold for caesium atoms is low $\left(E_{\mathrm{Ion}, \mathrm{I}}=3.89 \mathrm{eV}\right)$. Thus, usually a large fraction of caesium is singly ionized in the plasma of negative hydrogen ion sources for fusion[33]. Due to this high ionization degree the reaction rate of recombining processes is high and the caesium ion has a high relevance for populating the excited states. The threshold for removing a second electron is comparatively high $\left(E_{\mathrm{Ion}, \mathrm{II}}=23.16 \mathrm{eV}\right)$ and hence, the doubly charged positive caesium ion can be neglected.

Indicated in figure 1 by arrows are the most probable optical transitions 
Table 1: States of the caesium atom implemented to the CR model for caesium.

\begin{tabular}{lll}
\hline State & J & Energy $[\mathrm{eV}]$ \\
\hline $6^{2} \mathrm{~S}$ & $1 / 2$ & 0.0000 \\
$6^{2} \mathrm{P}$ & $1 / 2$ & 1.3861 \\
& $3 / 2$ & 1.4548 \\
$5^{2} \mathrm{D}$ & $3 / 2$ & 1.7979 \\
& $5 / 2$ & 1.8100 \\
$7^{2} \mathrm{~S}$ & $1 / 2$ & 2.2984 \\
$7^{2} \mathrm{P}$ & $1 / 2$ & 2.6989 \\
& $3 / 2$ & 2.7214 \\
$6^{2} \mathrm{D}$ & $3 / 2$ & 2.8010 \\
& $5 / 2$ & 2.8063 \\
$8^{2} \mathrm{~S}$ & $1 / 2$ & 3.0153 \\
$8^{2} \mathrm{P}$ & $1 / 2$ & 3.1879 \\
& $3 / 2$ & 3.1981 \\
$7^{2} \mathrm{D}$ & $3 / 2$ & 3.2299 \\
& $5 / 2$ & 3.2325 \\
Ion & & 3.8944 \\
\hline
\end{tabular}

$\left(A_{i k}>10^{4} \mathrm{~s}^{-1}\right)$ in the wavelength range between $400 \mathrm{~nm}$ and $900 \mathrm{~nm}$ originating from the excited states taken into account by the CR model. Amongst these are all transitions typically used for OES at caesium (the most prominent transition is the one at $852.1 \mathrm{~nm}$ ).

The following reaction types are implemented to the CR model: spontaneous emission, electron collision excitation and de-excitation, electron collision ionization, three-body and radiative recombination of $\mathrm{Cs}^{+}$and mutual neutralization of $\mathrm{Cs}^{+}$with $\mathrm{H}^{-}$. These reaction types are summarized in table 2. In order to enable implementing mutual neutralization, the list of states and species included in the model is extended by the negative hydrogen ion.

The caesium ground state density in the low-temperature, low-pressure plasma of the ion sources under investigation usually is lower as or around $10^{15} \mathrm{~m}^{-3}$, resulting in a high mean free path of photons. Since additionally the intensity of the photon field is low, processes like self-absorption due to optical thickness of emission lines[34] or photo ionization can be neglected. This is in contrast to the model presented in [25], developed for investigating neutral resonant ionization in a high-intensity caesium sputter source with a much higher ground state density $\left(n_{\mathrm{Cs}} \approx 10^{20} \mathrm{~m}^{-3}\right)$.

No other heavy particle collisions besides mutual neutralization are taken into account by the present CR model: based on rate constants from [42] it 
Table 2: Reaction types implemented to the CR model for caesium.

\begin{tabular}{llll}
\hline Process & Reaction & Ref. & Modification based on \\
\hline Excitation by $\mathrm{e}^{-}$collision & $\mathrm{Cs}(i)+\mathrm{e}^{-} \rightarrow \mathrm{Cs}(j>i)+\mathrm{e}^{-}$ & {$[35,36]$} & {$[37,38]$} \\
De-excitation by e $\mathrm{e}^{-}$collision & $\mathrm{Cs}(i)+\mathrm{e}^{-} \rightarrow \mathrm{Cs}(j<i)+\mathrm{e}^{-}$ & {$[35,36]$} & {$[37,38]$, detailed balance } \\
Spontaneous Emission & $\mathrm{Cs}(i) \rightarrow \mathrm{Cs}(j<i)+h \nu$ & {$[32,39]$} & \\
Ionization & $\mathrm{Cs}(i)+\mathrm{e}^{-} \rightarrow \mathrm{Cs}+2 \mathrm{e}^{-}$ & & {$[38]$} \\
Recombination of $\mathrm{Cs}^{+}$ & $\mathrm{Cs}^{+}+2 \mathrm{e}^{-} \rightarrow \mathrm{Cs}(j)+\mathrm{e}^{-}$ & & {$[38]$, Saha equation } \\
& $\mathrm{Cs}^{+}+\mathrm{e}^{-} \rightarrow \mathrm{Cs}(j)+h \nu$ & {$[40]$} & \\
Mutual neutralization & $\mathrm{Cs}^{+}+\mathrm{H}^{-} \rightarrow \mathrm{Cs}(j)+\mathrm{H}$ & {$[41]$} & \\
\hline
\end{tabular}

can be estimated that the reaction rates for associative ionization ( $\mathrm{Cs}+\mathrm{Cs} \rightarrow$ $\left.\mathrm{Cs}_{2}^{+}+\mathrm{e}^{-}\right)$, Penning ionization $\left(\mathrm{Cs}+\mathrm{Cs} \rightarrow \mathrm{Cs}^{+}+\mathrm{Cs}\left(6^{2} \mathrm{~S}\right)+\mathrm{e}^{-}\right)$and energy pooling of excited states are several orders of magnitude smaller compared to the rates for electron collision excitation and ionization (from the ground state) and for spontaneous emission. The same argument holds for ion pair formation taking place in collisions of excited caesium atoms and hydrogen atoms $\left(\mathrm{Cs}+\mathrm{H} \rightarrow \mathrm{Cs}^{+}+\mathrm{H}^{-}\right.$, cross sections from [43, 41]) and for ionization of excited caesium via charge exchange with protons $\left(\mathrm{Cs}+\mathrm{H}^{+} \rightarrow \mathrm{Cs}^{+}+\mathrm{H}\right.$, cross section for $6^{2} \mathrm{P}$ from [44]).

Taking into account the reaction types implemented to the model, the population density $n_{p}$ of the excited states $p$ of the caesium atom is described by the following rate equation:

$$
\begin{array}{r}
\frac{\mathrm{d} n_{p}}{\mathrm{~d} t}=\sum_{q>p} A_{q p} n_{q}-\sum_{q<p} A_{p q} n_{p}+ \\
+n_{\mathrm{e}}\left(\sum_{q \neq p} X_{q p} n_{q}-\sum_{q \neq p} X_{p q} n_{p}+\left(X_{\mathrm{Rad}, p}+X_{3 \text { body }, p} n_{\mathrm{e}}\right) n\left(\mathrm{Cs}^{+}\right)-X_{\mathrm{Ion}, p} n_{p}\right)+ \\
n\left(\mathrm{Cs}^{+}\right) n\left(\mathrm{H}^{-}\right) X_{\mathrm{MN}, p},
\end{array}
$$

where $q$ are all other excited states. $A_{q p}$ and $A_{p q}$ are the transition probabilities for spontaneous emission from $q$ to $p$ and $p$ to $q$, respectively. $X_{q p}$ and $X_{p q}$ are the rate coefficients for excitation or de-excitation by electron collisions. $X_{\mathrm{Rad}, p}$ and $X_{3 \mathrm{body}, p}$ are the rate coefficients for excitation by radiative and three-body recombination of the ion with density $n\left(\mathrm{Cs}^{+}\right)$and $X_{\text {Ion }, p}$ is the rate coefficient for ionization of the state $p . X_{\mathrm{MN}, p}$ is the rate coefficient for excitation of the state $p$ by mutual neutralization of $\mathrm{Cs}^{+}$with $\mathrm{H}^{-}$and $n\left(\mathrm{H}^{-}\right)$the negative hydrogen ion density.

The solver Yacora is applied in order to solve the resulting non-linear sys- 
tem of coupled ordinary differential rate equations. As initial condition for the integration process, the ground state density as well as the density of the singly charged caesium ion and the negative hydrogen ion are set (and fixed) while the population densities of all excited states are initially set to zero. This approach is based on the fact that the ground state density and the ion densities depend mainly on transport processes and equilibrium is reached on a much slower timescale (between milliseconds and microseconds) compared to the population densities of the electronically excited states (which are mainly determined by collisions with electrons or heavy particles and radiation, reaching equilibrium on the timescale of nanoseconds)[45]. Thus, the densities of the ground state and the ions can be treated as quasi-constant during calculating the population densities of the excited states.

\subsection{Probabilities for reactions interconnecting the energy levels of caesium}

The 15 states of the caesium atom implemented to the CR model (see table 1) consist of nine electronic states, six of which (the states with orbital angular momentum quantum number $L \neq 0$ ) split up due to different total angular momentum quantum numbers $J$. While the reaction probabilities available in the literature for spontaneous emission cover all optically allowed transitions interconnecting these 15 states (see section 2.2.1), almost all available cross sections for collisional processes neglect the fine-structure splitting.

Thus, the following procedure is implemented to the CR model: For collisional excitation and de-excitation as well as for ionization, recombination and mutual neutralization the fine structure is not taken into account. For example, during the electron collision excitation from $6^{2} \mathrm{~S}$ to $7^{2} \mathrm{P}$ it is not distinguished between the two sub-states $7^{2} \mathrm{P}_{1 / 2}$ and $7^{2} \mathrm{P}_{3 / 2}$. The threshold energy of this excitation process is determined as the average energy of the sub-states. However, for determining the spontaneous emission rate the total population density of $7^{2} \mathrm{P}$ is distributed amongst $7^{2} \mathrm{P}_{1 / 2}$ and $7^{2} \mathrm{P}_{3 / 2}$ according to the Boltzmann distribution defined by $T_{\mathrm{e}}$. For these two sub-states then the appropriate transition probabilities are used. The choice of a Boltzmann distribution is justified by a high probability for electron collisions thermalizing the population densities of the sub-states resulting from the fine-structure splitting, caused by the very small energy difference between these states.

Output of the model are the calculated population densities for the nine implemented electronic states (i.e. neglecting the fine-structure splitting). 
These population densities can either be used directly or be split up subsequently (using again a Boltzmann distribution) in order to obtain the population densities of the sub-states caused by the fine-structure splitting. Either way, using the appropriate transition probabilities for spontaneous emission from and to the sub-states during the calculation process increases the accuracy of the modeling results compared to models neglecting the fine-structure splitting.

The reaction probabilities (transition probabilities for spontaneous emission, cross sections for the collisional processes) for the different reaction types included in the model have been taken - as far as possible - from the literature. The respective references are given in the third column of table 2 . The cross sections for most of the collisional processes have been corrected or extended in order to obtain a comprehensive set of all input data needed for the CR model. Given in the fourth column of table 2 are references and methods used for modifying the literature data. The data from the literature and the performed modifications for all reaction types are described in the following sections. Additionally, the necessity for performing these modifications is explained in detail.

\subsubsection{Spontaneous emission}

As far as possible, transition probabilities from [32] are used. Although this comprehensive list contains all caesium emission lines between the vacuum ultraviolet wavelength range and the mid-wavelength infrared, it does not give transition probabilities for all these lines. Thus, the set of transition probabilities for the $\mathrm{CR}$ model was completed by adding values taken from [39].

\subsubsection{Electron collision excitation and de-excitation}

Available in the literature is a multitude of theoretically[35, 46, 47, 48, 49, $36,50,51,52]$ and experimentally determined $[37,53,54,55]$ electron collision excitation cross sections for caesium. Most of these data is for excitation from the ground state $6^{2} \mathrm{~S}$ to the first two or three excited states. For excitation to energetically higher states and also for collisions interconnecting different excited states the data is scarce.

For preparing the input data needed for the CR model it was necessary to choose and assemble cross sections from the different data sources. The available cross sections show large discrepancies, as illustrated in figure 2 for excitation from $6^{2} \mathrm{~S}$ to the first excited state $6^{2} \mathrm{P}$. Several of the theoretical 


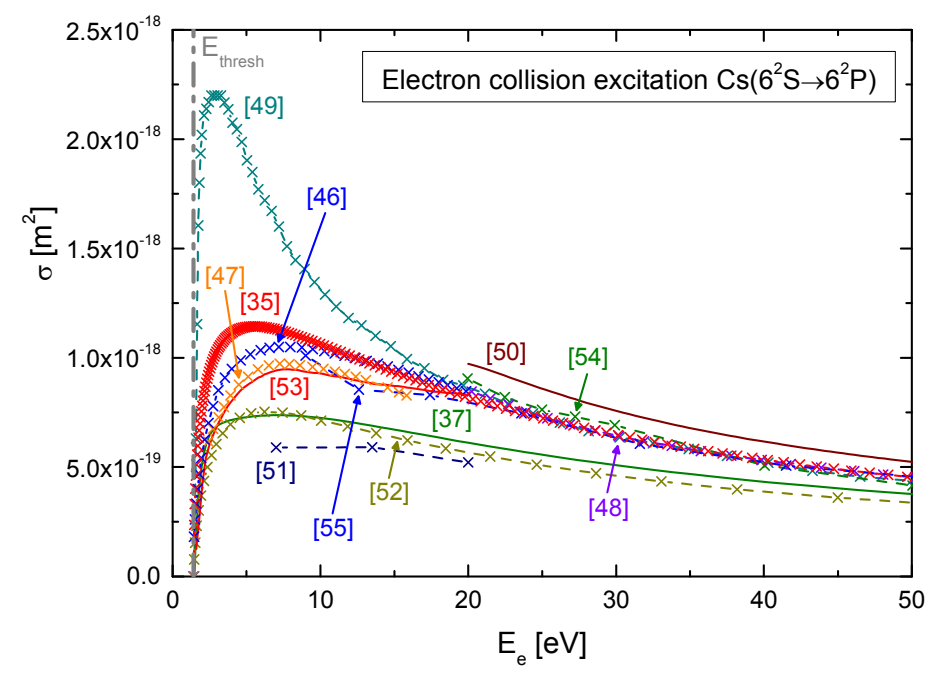

Figure 2: Cross sections from different data sources for electron collision excitation from $6^{2} \mathrm{~S}$ to $6^{2} \mathrm{P}$. The large discrepancies occurring close to the threshold region can be significantly reduced by applying equation 2 to the Born or Bethe cross sections.

cross sections are based on the Born or the Bethe method. In [37] it was shown that close to the threshold energy excitation cross sections calculated by these methods are generally by a factor of two to three higher compared to measured values - indicating a large error bar of the calculations in this energy range. Suggested in [37] is the following simple empirical formula for correcting such cross sections for alkali metals in the low-energy region close to the threshold:

$$
\sigma_{\text {corr }}=\left(1-\sqrt{\frac{E_{\text {thresh }}}{E_{\mathrm{e}}}}\right) \sigma_{\text {Born/Bethe }},
$$

where $E_{\mathrm{e}}$ is the electron energy and $E_{\text {thresh }}$ the excitation threshold. By applying this correction scheme, the discrepancies between the data taken from different sources shown in figure 2 can be reduced significantly.

Thus, for implementing electron collision excitation cross sections to the $\mathrm{CR}$ model the most complete literature source was chosen: Born cross sections from [35] for excitation from $6^{2} \mathrm{~S}, 6^{2} \mathrm{P}$ and $5^{2} \mathrm{D}$ to the excited states up to $6^{2} \mathrm{D}$ are used, corrected by applying equation 2 .

Excitation of states energetically higher than $6^{2} \mathrm{D}$ is not treated in [35]. 


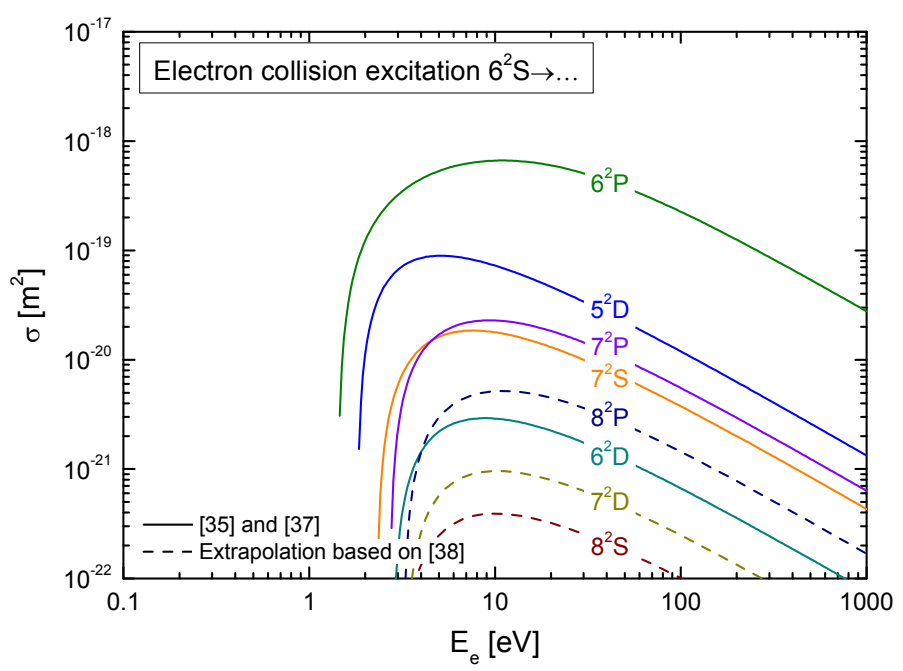

Figure 3: Cross sections for electron collision excitation from the state $6^{2} \mathrm{~S}$ in the caesium atom from [35] (corrected by applying equation 2) and the result of the performed extrapolation to energetically higher states based on [38].

Thus, the cross sections for electron collision excitation from $6^{2} \mathrm{~S}, 6^{2} \mathrm{P}$ and $5^{2} \mathrm{D}$ to $8^{2} \mathrm{~S}, 8^{2} \mathrm{P}$ and $7^{2} \mathrm{D}$ have been determined by applying an extrapolation scheme based on the Gryzinski method[38]: the cross section for excitation of a certain state is deduced from the known corrected Born cross section for excitation of an energetically lower lying state with the same orbital angular momentum, taking into account the different threshold energies. In order to estimate the error of this extrapolation scheme, the cross section for the electron collision excitation process $6^{2} \mathrm{~S} \rightarrow 7^{2} \mathrm{P}$ is extrapolated from the corrected Born data for $6^{2} \mathrm{~S} \rightarrow 6^{2} \mathrm{P}$ and compared with the available corrected Born cross section from [35]: the deviation between the two cross sections is below a factor of two, increasing slightly only close to the threshold.

Cross sections from [36], corrected by applying equation 2, are additionally used for collisions interconnecting excited states above $5^{2} \mathrm{D}$ and for some of the inverse reactions (electron collision de-excitation). If the cross section for a specific electron collision de-excitation process is not available in the literature, it is deduced from the corresponding electron collision excitation cross section using the detailed balance principle.

The described data represents the best set of electron collision excitation cross sections available for caesium up to now. The cross sections for excita- 


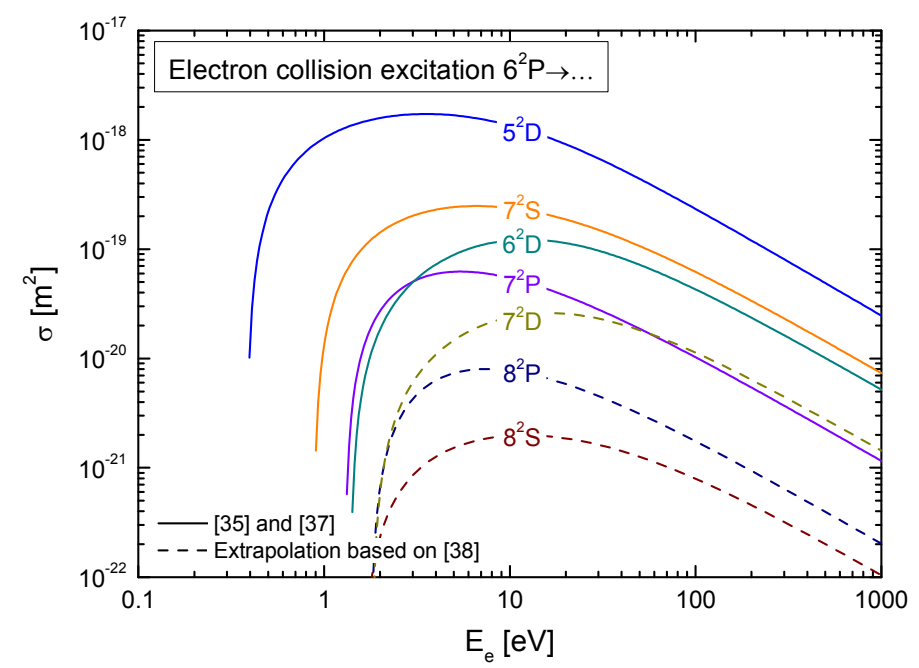

Figure 4: Cross sections for electron collision excitation from the state $6^{2} \mathrm{P}$ in the caesium atom from [35] (corrected by applying equation 2) and the result of the performed extrapolation to energetically higher states based on [38].

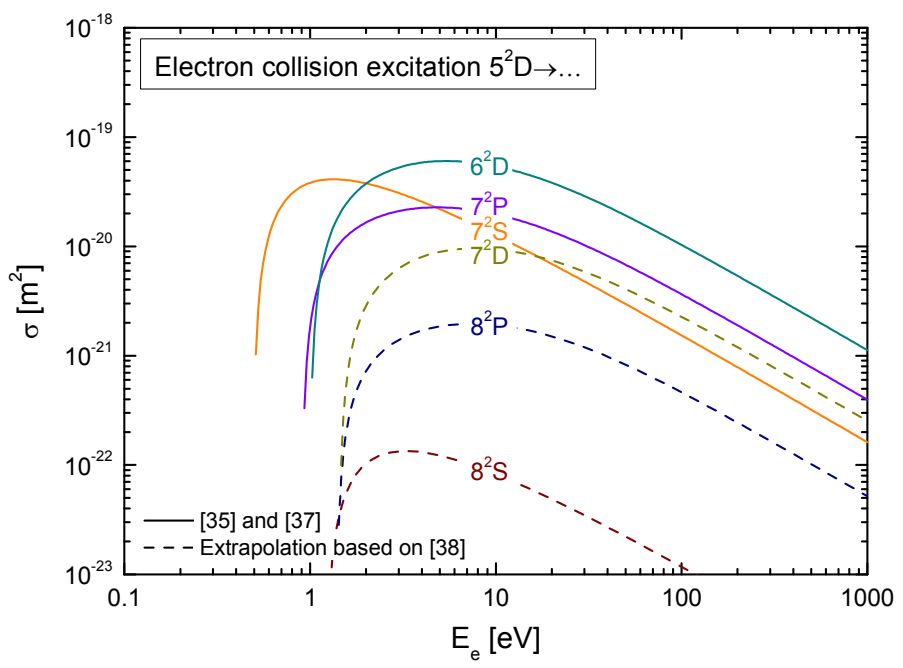

Figure 5: Cross sections for electron collision excitation from the state $5^{2} \mathrm{D}$ in the caesium atom from [35] (corrected by applying equation 2) and the result of the performed extrapolation to energetically higher states based on [38]. 
tion from the three (energetically lowest) states $6^{2} \mathrm{~S}, 6^{2} \mathrm{P}$ and $5^{2} \mathrm{D}$ are shown in figures 3,4 and 5 .

\subsubsection{Electron collision ionization and recombining processes}

Theoretically and experimentally determined cross sections for electron collision ionization of caesium are available in the literature for the ground state $6^{2} \mathrm{~S}$ and a few excited states only[56, 57, 58, 59]. This set of cross sections is not sufficient for being implemented to the CR model.

In order to generate a consistent set of cross sections, new cross sections for ionization of the excited states of caesium were calculated using the Gryzinski method[38]. The Gryzinski method is a classical method taking into account only the transition energy (and in molecules additionally the Franck-Condon factor) between the corresponding states. Thus, usually the accuracy of excitation cross sections determined purely by the Gryzinski method is assumed to be relatively low - the uncertainty is within a factor of 2-3[60], close to the threshold it can be even higher.

However, in [61] it was shown that the accuracy of ionization cross sections produced using the Gryzinski method is surprisingly high: Gryzinski cross sections for ionization of the ground state $X^{1} \Sigma_{g}^{+}(v=0)$ of the hydrogen molecule are in almost perfect agreement with the latest and most reliable experimental results. Reason for this good agreement is that during ionization the outermost valence electron is removed completely. This reaction can be described much more accurately by classical methods than the shift of the valence electron from one shell to a specific other shell taking place during an excitation reaction.

For caesium a similar observation is made as for hydrogen: the newly calculated ionization cross sections for the caesium atom are shown in figure 6 , together with the most recent theoretical cross section for ionization of the ground state $6^{2} \mathrm{~S}$ available in literature[59] (calculated by the convergent close-coupling (CCC) method). The agreement of the CCC cross section and the Gryzinski result is very good - especially in the energy region close to the threshold.

The rate coefficient $X_{3 \text { body }, p}$ for three-body recombination into the excited state $p$ is determined from the ionization rate coefficient $X_{\mathrm{Ion}, p}$ by applying the detailed balance principle and the Saha equation:

$$
X_{\text {3body }, p}=\frac{1}{2} \frac{g_{\mathrm{Cs}, p}}{g_{\mathrm{Cs}}}\left(\frac{2 \pi \hbar^{2}}{m_{\mathrm{e}} k_{\mathrm{B}} T_{\mathrm{e}}}\right)^{3 / 2} \exp \left(\frac{E_{\mathrm{Ion}, p}}{k_{\mathrm{B}} T_{\mathrm{e}}}\right) \cdot X_{\text {Ion }, p},
$$




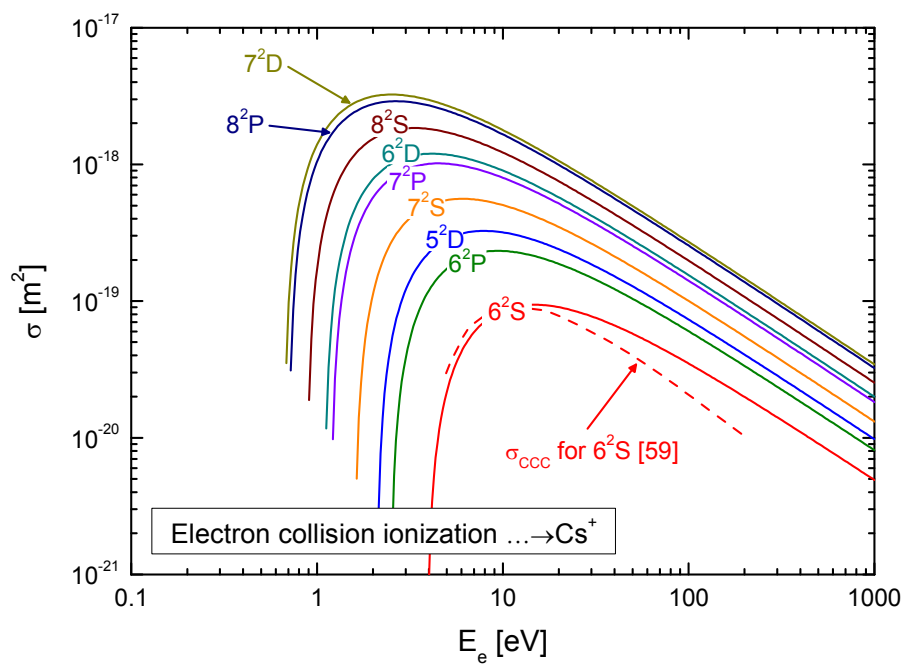

Figure 6: Cross sections for ionization of the first excited states in the caesium atom calculated by the Gryzinski method[38] and the CCC method.

where $g_{\mathrm{Cs}, p}$ and $g_{\mathrm{Cs}^{+}}$are the statistical weights of the caesium atom in the excited state $p$ and of the caesium ion, respectively. $E_{\mathrm{Ion}, p}$ is the ionization threshold of the state $p$.

State-resolved cross sections for radiative recombination are given in [40], but for five different electron energies only (between $E_{\mathrm{e}}=0.07 \mathrm{eV}$ and $1.09 \mathrm{eV})$. Additionally given in [40] are the respective rate coefficients for five values of the electron temperature (between $T_{\mathrm{e}}=0.09 \mathrm{eV}$ and $0.29 \mathrm{eV}$, based on a Maxwell electron energy distribution function).

In order to facilitate implementation of the cross sections from [40] to the CR model a cubic polynomial fit (in a log-log scale) for electron energies between $E_{\mathrm{e}}=0.07 \mathrm{eV}$ and $1.09 \mathrm{eV}$ and a linear extrapolation (again in the log-log scale) to electron energies between $0.01 \mathrm{eV}$ and $100 \mathrm{eV}$ have been performed. The validity of this fitting and extrapolation procedure was checked by convolving the extrapolated cross sections with a Maxwell electron energy distribution function to rate coefficients and comparing these with the five rate coefficients additionally given in [40]. The agreement for most states is quite good (the deviations are around or well below 10\%). For the two states $7^{2} \mathrm{~S}$ and $8^{2} \mathrm{~S}$ the deviation is higher but it decreases quickly with the electron temperature (from around $40 \%$ for $T_{\mathrm{e}}=0.09 \mathrm{eV}$ to around $15 \%$ for $T_{\mathrm{e}}=0.29 \mathrm{eV}$, i.e. for electron temperatures around $1 \mathrm{eV}$ as present in the ion 


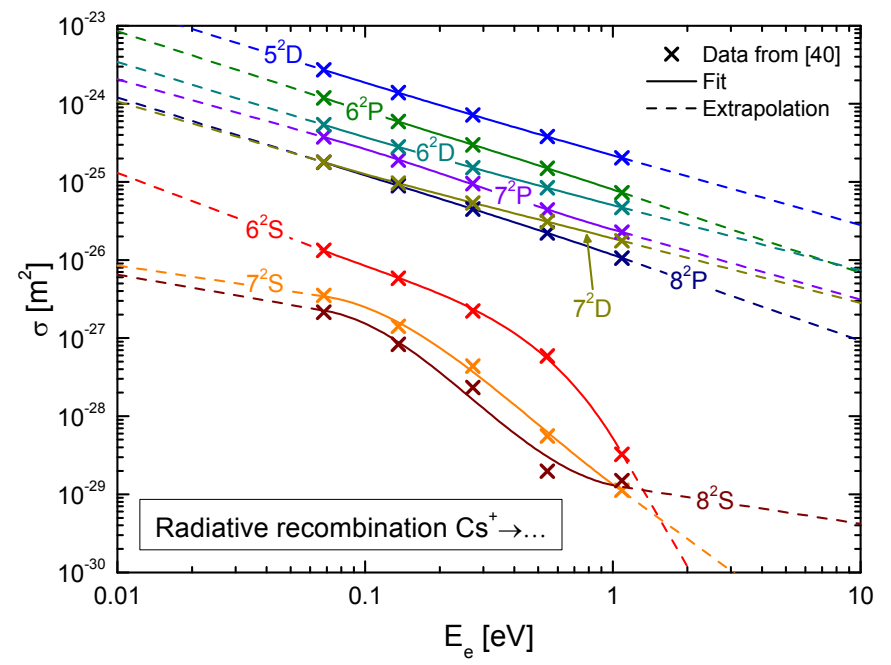

Figure 7: Cross sections for radiative recombination of the caesium ion into the first excited states in the caesium atom. Shown are the values from [40], the result of the performed fit and the extrapolation to high and low electron energies.

sources under investigation even smaller deviations can be expected).

The cross sections for radiative recombination implemented to the CR model are shown - together with the raw data from [40] - in figure 7.

\subsubsection{Mutual neutralization with negative hydrogen ions}

Mutual neutralization of singly charged positive caesium ions and negative hydrogen ions produces atoms (hydrogen and caesium) in the respective ground state or in an excited state. Two different sets of mutual neutralization cross sections are available in the literature: the first one[26], calculated using the Landau-Zener technique, consists of cross sections for generation of caesium atoms in the ground state $6^{2} \mathrm{~S}$, the excited states $6^{2} \mathrm{P}$, $7^{2} \mathrm{~S}$ and $7^{2} \mathrm{P}$ and for collision energies between $10^{-2} \mathrm{eV}$ and $3.1 \cdot 10^{3} \mathrm{eV}$ (in the center-of-mass frame). The second data set, presented in [43], is based on the perturbed-stationary-state method with electron translation factors. Cross sections are given for the states $6^{2} \mathrm{~S}, 6^{2} \mathrm{P}, 5^{2} \mathrm{D}$ but for three values of the collision energy only $\left(E_{\mathrm{CM}}=0.1 \mathrm{keV}, 1.0 \mathrm{keV}\right.$ and $10.0 \mathrm{keV}$, also in the center-of-mass frame).

The mutual neutralization cross sections from [26] and [43] are shown in figure 8. Regarding the final states, none of the two data sets is complete: 


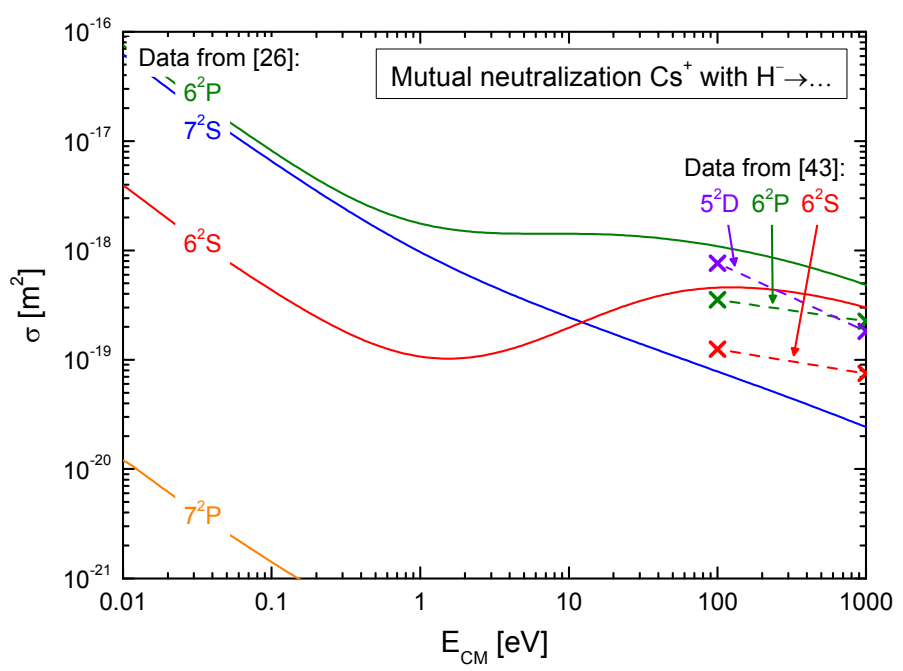

Figure 8: Cross sections available in the literature for generation of excited states in the caesium atom by mutual neutralization of $\mathrm{Cs}^{+}$with $\mathrm{H}^{-}$.

In the data from [26], the relevant channel resulting in caesium atoms in the state $5^{2} \mathrm{D}$ is missing while in [43] no cross section for excitation of $7^{2} \mathrm{~S}$ is given. The channel ending up in a caesium atom excited to $7^{2} \mathrm{P}$, also missing in [43], can be neglected due to its small cross section and consequently small reaction rate. Generating the needed input data for the CR model by simply merging the available cross sections is not possible since the data from [43] is for collision energies above $100 \mathrm{eV}$ only - much higher than the collision energies in the considered low-temperature, low-pressure hydrogen-caesium plasmas (up to a few eV at maximum).

Instead, data from [41] to be published is used for implementing mutual neutralization to the CR model. These cross sections have been calculated using the branching classical trajectory method[62]. According to [62] it can be assumed that due to a more comprehensive treatment of the mutual neutralization process the results of this methods are more accurate compared to Landau-Zener calculations. Cross sections are available for the final states $6^{2} \mathrm{~S}, 6^{2} \mathrm{P}, 7^{2} \mathrm{~S}$ and $5^{2} \mathrm{D}$ and for collision energies starting at $0.01 \mathrm{eV}$ (in the center-of-mass frame). For high energies $\left(E_{\mathrm{CM}}>250 \mathrm{eV}\right)$ and the final states $6^{2} \mathrm{~S}, 6^{2} \mathrm{P}, 7^{2} \mathrm{~S}$ these cross sections are comparable with the ones from [26]; for lower energies large deviations (up to a factor of 50) between the two data sets exist. 


\subsection{Dependence of population densities on the plasma parameters}

This section presents calculated population densities of the three excited states $6^{2} \mathrm{P}, 7^{2} \mathrm{P}$ and $7^{2} \mathrm{D}$. These are the upper states of seven of the most intense caesium emission lines $\left(6^{2} \mathrm{P}\right.$ : at $852.1 \mathrm{~nm}$ and $894.3 \mathrm{~nm}, 7^{2} \mathrm{P}$ : at $455.5 \mathrm{~nm}$ and $459.3 \mathrm{~nm}, 7^{2} \mathrm{D}$ : at $672.3 \mathrm{~nm}, 697.3 \mathrm{~nm}$ and $698.3 \mathrm{~nm}$, see also figure 1).

Shown in figure 9 is the dependence of the calculated population densities on the electron temperature and the electron density. The ground state density $n\left(6^{2} \mathrm{~S}\right)$ has been set to $10^{15} \mathrm{~m}^{-3}$ and $n\left(\mathrm{Cs}^{+}\right)$to $9 \cdot 10^{15} \mathrm{~m}^{-3}$. These values represent typical parameters in the ion sources under investigation (see section 3.2). The negative hydrogen ion density is set to zero, i.e. the mutual neutralization is switched-off for these calculations. For the electron energy a Maxwell distribution function is used.

Excitation by electron collision (mainly directly from the ground state $6^{2} \mathrm{~S}$ ) is the dominant excitation channel for the complete range of investigated $T_{\mathrm{e}}$ and $n_{\mathrm{e}}$. Thus, the dependence of the population densities on $T_{\mathrm{e}}$ is mainly determined by the well known behavior of electron collision excitation rate coefficients: a strong increase for low $T_{\mathrm{e}}$, followed by a steady decrease of the gradient (figure 9a). This decrease of the gradient starts at a relatively low electron temperature $\left(T_{\mathrm{e}} \approx 1.5 \ldots 2 \mathrm{eV}\right)$. Reason are the low threshold energies for direct electron collision excitation from the ground state (see table 1). For high electron temperatures $\left(T_{\mathrm{e}}>20 \ldots 60 \mathrm{eV}\right.$, also depending on the electron density) the population densities decrease with the temperature (not shown here). For low electron densities the calculated population densities approach the results of the corona models for the three states. The densities increase nearly linear with the electron density (figure 9b). This is as well caused by the prevailing role of electron collisions for exciting the three states.

The negative hydrogen ion density in the low-temperature, low-pressure hydrogen-caesium plasmas of negative ion sources based on surface production can reach values up to slightly above $10^{17} \mathrm{~m}^{-3}[63,64,65]$. Figure 10 shows - calculated for $T_{\mathrm{e}}=2 \mathrm{eV}$ and $n_{\mathrm{e}}=10^{17} \mathrm{~m}^{-3}$ - population densities of $6^{2} \mathrm{P}, 7^{2} \mathrm{P}$ and $7^{2} \mathrm{D}$ for negative hydrogen ion densities between $10^{14} \mathrm{~m}^{-3}$ and $10^{18} \mathrm{~m}^{-3}$.

As can be expected, for low negative hydrogen ion densities the population densities approach the results for without negative ions. With increasing $n\left(\mathrm{H}^{-}\right)$the population densities of all three states increase, but to a different extent: since $6^{2} \mathrm{P}$ is the final state of one of the mutual neutralization 

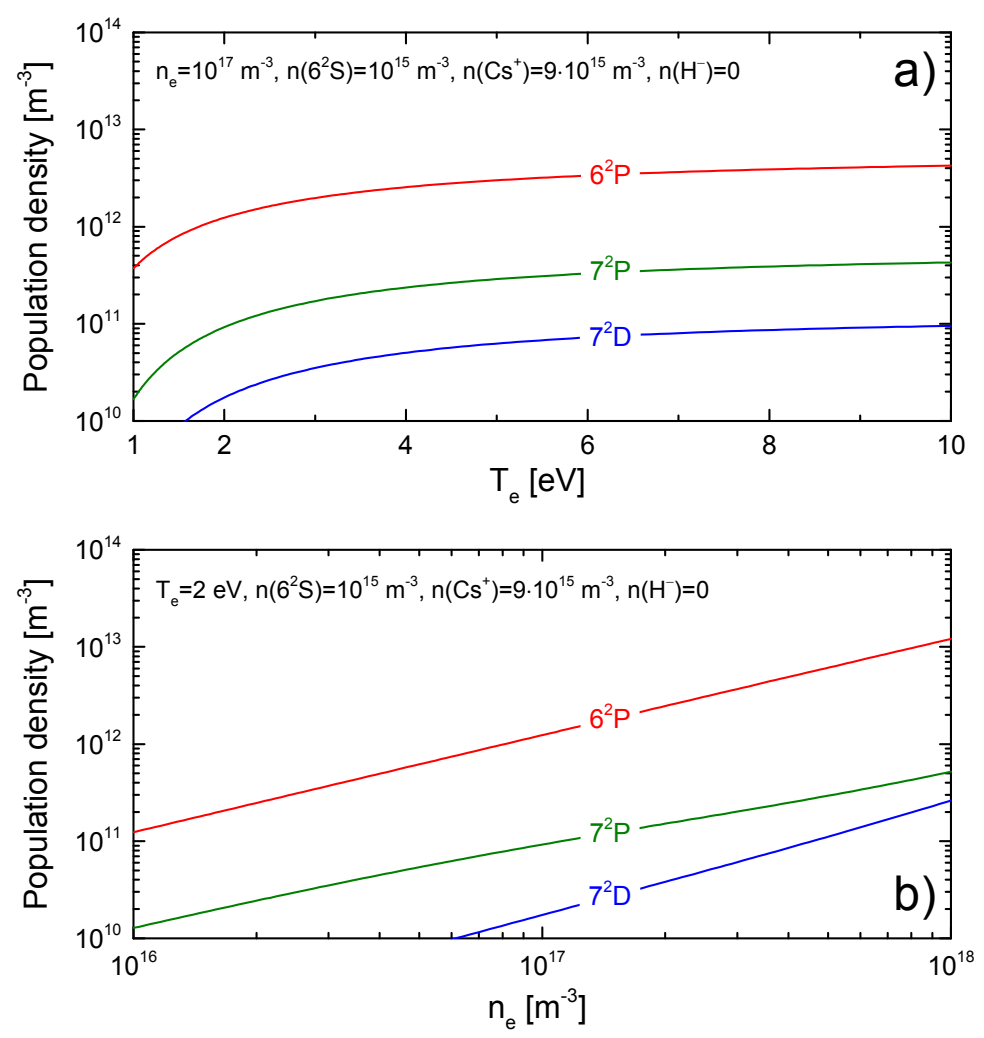

Figure 9: Population densities of the three states $6^{2} \mathrm{P}, 7^{2} \mathrm{P}$ and $7^{2} \mathrm{D}$ in the caesium atom calculated using the CR model. a) Scan of the electron temperature $T_{\mathrm{e}}$. b) Scan of the electron density $n_{\mathrm{e}}$. 


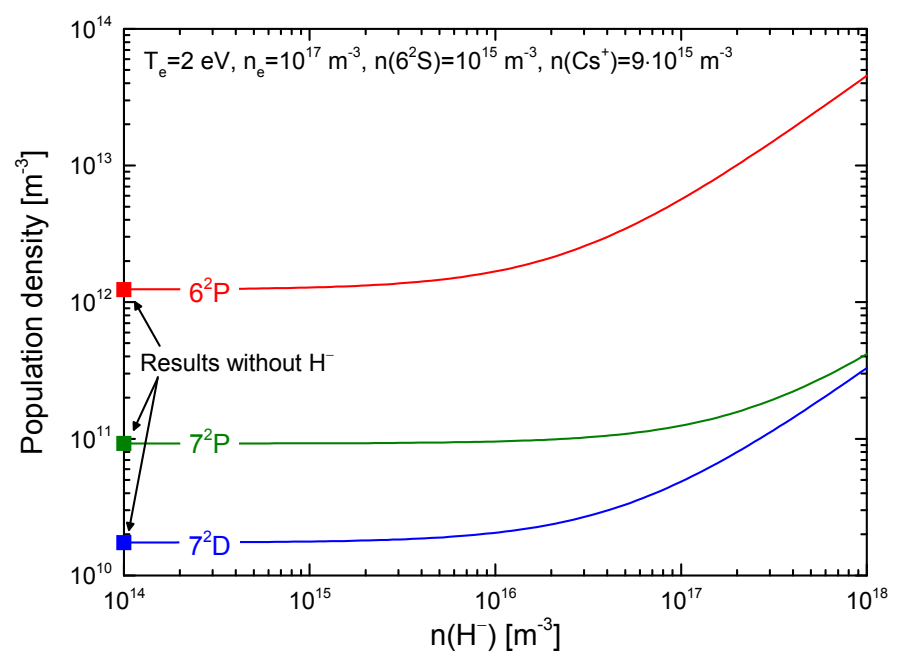

Figure 10: Population densities of the three states $6^{2} \mathrm{P}, 7^{2} \mathrm{P}$ and $7^{2} \mathrm{D}$ in the caesium atom in dependence on the density of negative hydrogen ions (for $n\left(\mathrm{Cs}^{+}\right)=9 \cdot n(\mathrm{Cs})$ ).

channels (see section 2.2.4) its population density is directly increased with increasing $n\left(\mathrm{H}^{-}\right)$. A slightly weaker increase of $n\left(7^{2} \mathrm{D}\right)$ with $n\left(\mathrm{H}^{-}\right)$can be seen in the parameter range under investigation, while $n\left(7^{2} \mathrm{P}\right)$ is much less affected. The increase of the two latter population densities is an indirect effect: first, the population densities of the final states of mutual neutralization $\left(6^{2} \mathrm{P}, 7^{2} \mathrm{~S}\right.$ and $\left.5^{2} \mathrm{D}\right)$ are increased. Then, these increased population densities are distributed amongst the other excited states by means of reactions interconnecting the excited states (mainly electron collisions). The additional excitation rate for $7^{2} \mathrm{P}$ and $7^{2} \mathrm{D}$ caused by these interconnecting reactions is roughly comparable. However, the reaction rate for electron collision excitation from the ground state (the main excitation channel in the case without $\mathrm{H}^{-}$) is significantly smaller for $7^{2} \mathrm{D}$ compared to $7^{2} \mathrm{P}$ (caused by the higher excitation threshold energy). Consequently, the influence of the additional excitation caused by mutual neutralization on the total excitation rate of $7^{2} \mathrm{D}$ is higher than for $7^{2} \mathrm{P}$.

A direct result of the increased excited state population densities is an increased intensity of the spontaneous emission lines originating from these states. Thus, OES is an appropriate tool to verify the strong influence of mutual neutralization predicted by the CR model. 


\section{Comparison with experimental results}

\subsection{The experiment and the performed measurements}

Population densities of excited caesium states calculated by the CR model are compared with OES results taken at the low-temperature, low-pressure plasma of the IPP prototype negative hydrogen ion source at the BATMAN test facility[12, 28]. Since 2007 the reference design for the ITER NBI sources is based on this source[66].

Figure 11 shows a schematic view of the prototype source: the plasma is generated in a cylindrical driver by inductive $\mathrm{RF}$ coupling $\left(P_{\mathrm{RF}, \max } \approx 90 \mathrm{~kW}\right)$. The plasma then expands into the expansion region where it is cooled (from $T_{\mathrm{e}} \gtrsim 10 \mathrm{eV}$ to $\approx 1 \mathrm{eV}$ at $n_{\mathrm{e}} \approx 10^{17} \mathrm{~m}^{-3}$ ) by means of a magnetic filter field (of some $\mathrm{mT}$ ). The filter field is generated by rods of permanent magnets embedded into an external frame[67]. The dominant component of the filter field points into the horizontal direction.

The most important converter surface for generating negative hydrogen ions is the surface of the plasma grid $(\mathrm{PG})$, the first grid of a multi aperture multi-grid extraction system. The amount of co-extracted electrons, already reduced by the filter field, can be further decreased by a positive bias potential applied to the PG with respect to the source body and a so-called bias plate. The caesium injection system is attached to the upper part of the source back plate.

A complex plasma region (extent in axial direction: several centimeters) close to the PG - the so-called boundary layer[68] - evolves in an interplay of positive ions and electrons generated (predominantly) in the hot driver plasma, caesium atoms and caesium ions coming from the injection system and from reservoirs at the walls, negative ions generated at the $\mathrm{PG}$, the magnetic filter field and the PG bias. Almost all physical aspects relevant for producing a uniform and stable negative hydrogen ion beam take place in this boundary layer[69].

For investigating the boundary layer several diagnostic ports are available providing lines of sight (LOS) parallel to the PG. Within the scope of the present investigations two horizontal LOS with $2.0 \mathrm{~cm}$ distance to the PG are used for OES: one (XR1) in the upper part of the ion source and another (XL1) in the lower part. Both LOS are shown in figure 11.

Reason for using two different horizontal LOS is that during previous campaigns strong vertical non-uniformities of the plasma in front of the extraction system have been observed[70], caused by a plasma drift. This drift 


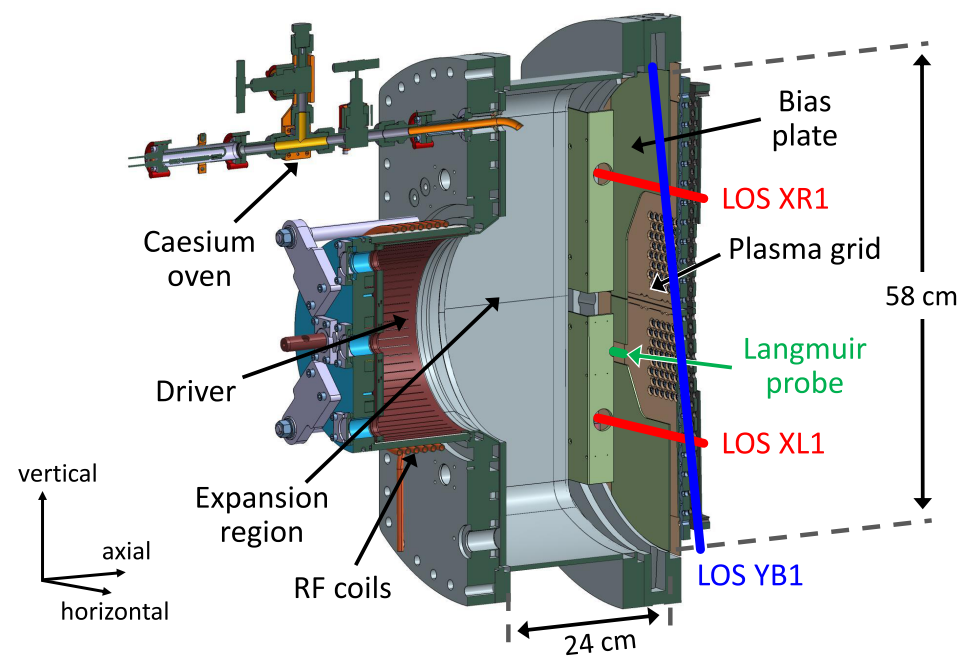

Figure 11: Schematic view of the IPP prototype negative hydrogen ion source, the two horizontal lines of sight (XR1 and XL1) used for the performed OES measurements, the vertical line of sight (YB1) of the laser absorption spectroscopy and one of the two Langmuir probes.

is induced by the horizontal filer field in interplay with axial potential gradients in the plasma. During the present measurements the magnetic field was used in the configuration "drift up", i.e. close to the PG a vertical shift of the bulk plasma towards the upper half of the ion source takes place.

The intensity of six emission lines originating from the excited states $6^{2} \mathrm{P}, 7^{2} \mathrm{P}$ and $7^{2} \mathrm{D}$ along these two LOS was determined by means of an absolutely calibrated spectrometer (ACTON SpectraPro-750i). Due to the limited wavelength range detectable by the spectrometer, six successive and identical pulses were performed and between each of these six pulses the central wavelength of the spectrometer was changed. For each of the six lines a sufficient intensity was detected, resulting in a relatively small error bar of the measurements $(<10 \%)$. The population densities of the three excited states were deduced by dividing the measured line emissivities by the according Einstein coefficient and summing up - for each of the three states - the results for the two respective sub-states resulting from the finestructure splitting.

In order to check the predicted high relevance of mutual neutralization, these measured population densities are compared with results of the CR 
model. For each of the two LOS measurements for two different plasma regimes were performed: first, without bias current flowing to the PG, i.e. the PG potential was set to the floating potential. And second, with the bias potential optimized for reducing the co-extracted electron current without affecting too much the extracted negative ion current. The bias potential mainly influences the absolute value and the vertical distribution of the plasma density close to the PG (see section 3.2).

A prerequisite of performing $\mathrm{CR}$ model calculations is the knowledge of the plasma parameters $\left(T_{\mathrm{e}}, n_{\mathrm{e}}, n\left(6^{2} \mathrm{~S}\right), n\left(\mathrm{Cs}^{+}\right), n\left(\mathrm{H}^{-}\right)\right)$in the discharges under investigation. An overview of the plasma parameters used as input for the model is given in the following section.

\subsection{Plasma parameters in the IPP prototype source}

During the investigated discharges the local electron temperature and electron density have been determined by two Langmuir probes[70]. These probes are positioned at the vertical side walls of the ion source - one probe in the upper part of the source and the second in the lower part. The axial distance between the probe tips and the PG $\left(\Delta_{\text {axial }}=0.8 \mathrm{~cm}\right)$ and also the vertical distance from the center of the $\mathrm{PG}$ is smaller compared to the LOS used for OES - as can be seen in figure 11. Shown in the figure is only one of the probes (in the lower part of the source), another one is located in the upper part of the ion source close to the side wall cut away in the figure. The electron temperature was determined by the probes to be $2.0 \mathrm{eV}$ with bias and $2.1 \mathrm{eV}$ without bias, for both used LOS. The measured electron densities are between $2.8 \cdot 10^{16} \mathrm{~m}^{-3}$ and $10^{17} \mathrm{~m}^{-3}$ where $n_{\mathrm{e}}$ is always higher in the upper part of the source compared to the lower part. Without bias the ratio $n_{\text {e,upper }} / n_{\text {e,lower }}$ is 2.1 and it increases to 3.6 with bias. These observations can be explained by the upward vertical plasma drift mentioned before. The effect of this drift is increased by increasing the bias potential.

For the caesium ground state density $n\left(6^{2} \mathrm{~S}\right)$ a lower limit between 6 . $10^{14} \mathrm{~m}^{-3}$ and $7 \cdot 10^{14} \mathrm{~m}^{-3}$ has been determined during the present campaign by laser absorption spectroscopy[71] along a vertical LOS (YB1, see figure 11). These caesium densities are not directly comparable to the OES and CR model results since the vertical LOS averages - in contrast to the two horizontal LOS used for OES - the caesium densities in the upper and the lower part of the ion source. Laser absorption spectroscopy measurements based on the two LOS XR1 and XL1 performed during previous experimental campaigns indicate that due to the position of the caesium injection system 
(at the upper part of the source back plate) the caesium density in the upper part of the ion source usually is larger compared to the lower part. Typical values for the ratio $n\left(6^{2} \mathrm{~S}\right)_{\text {upper }} / n\left(6^{2} \mathrm{~S}\right)_{\text {lower }}$ determined by laser absorption spectroscopy during plasma pulses are between one and two. Depending on the caesium distribution as well as the direction and the strength of the vertical plasma drift sometimes also significantly higher values of this ratio are possible.

The other input parameters for the CR model have not been determined during the present experimental campaign, but typical values from previous measurements are used: by OES the ionization degree of caesium has been determined to be around $90 \%[33]$.

The density $n\left(\mathrm{H}^{-}\right)$of negative hydrogen ions close to the $\mathrm{PG}$ is taken from laser detachment [63] and cavity ring-down[64, 65] measurements: depending on the filling pressure, the applied RF power and the status of the caesium conditioning, $n\left(\mathrm{H}^{-}\right)$can reach values slightly higher than $10^{17} \mathrm{~m}^{-3}$. No measured upper/lower ratio of the negative ion density is available.

The described plasma parameters are used as starting point for an optimization procedure: for $T_{\mathrm{e}}$ and $n_{\mathrm{e}}$ deviations of up to $\pm 1 \mathrm{eV}$ and $\pm 15 \%$ from the measured values were allowed, respectively (representing the error bar of the probe results). The caesium ground state density $n\left(6^{2} \mathrm{~S}\right)$ and $n\left(\mathrm{H}^{-}\right)$are free parameters. The ratio of singly ionized caesium to caesium in its ground state is set to the fixed value 9.0. The obtainable degree of agreement between experimentally determined and calculated population densities is a measure for the validity of the CR model.

\subsection{Results of applying the CR model for calculating population densities}

Figure 12 shows in full symbols the population densities of the states $6^{2} \mathrm{P}$, $7^{2} \mathrm{P}$ and $7^{2} \mathrm{D}$ from OES for the four performed measurements. Shown in open squares are the results of the performed CR model calculations.

The input parameters used for these calculations are summarized in table 3. Taking mutual neutralization into account, for all measurements a good agreement between the population densities measured by OES and the CR model results is obtained.

The predicted influence of mutual neutralization on the population densities can clearly be seen: besides the best possible match of model to experiment figure 12 additionally shows (open circles) the result for the same set of input parameters, but calculated without mutual neutralization. While for the upper LOS (XR1) a good match of CR model results and measurements 


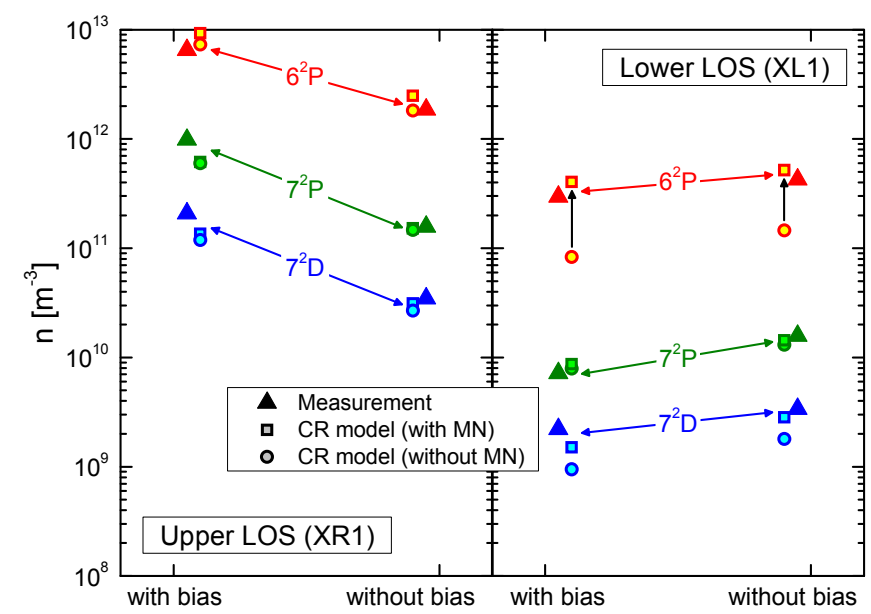

Figure 12: Comparison of experimentally determined population densities (full triangles) and the results of the CR model for the four performed measurements. Results of calculations with (open squares) and without mutual neutralization (open circles) are shown. The strong influence of mutual neutralization on the population density of $n\left(6^{2} \mathrm{P}\right)$ is indicated by vertical arrows.

Table 3: Plasma parameters used in order to reproduce the measured population densities by the $\mathrm{CR}$ model.

\begin{tabular}{lllll}
\hline Measurement set & $T_{\mathrm{e}}[\mathrm{eV}]$ & $n_{\mathrm{e}}\left[\mathrm{m}^{-3}\right]$ & $n\left(6^{2} \mathrm{~S}\right)\left[\mathrm{m}^{-3}\right]$ & $n\left(\mathrm{H}^{-}\right)\left[\mathrm{m}^{-3}\right]$ \\
\hline XR1, with bias & 2.5 & $1.0 \cdot 10^{17}$ & $4.5 \cdot 10^{15}$ & $1.0 \cdot 10^{16}$ \\
XR1, without bias & 2.2 & $8.7 \cdot 10^{16}$ & $1.5 \cdot 10^{15}$ & $1.0 \cdot 10^{16}$ \\
XL1, with bias & 2.0 & $2.8 \cdot 10^{16}$ & $2.4 \cdot 10^{14}$ & $3.0 \cdot 10^{16}$ \\
XL1, without bias & 2.0 & $4.2 \cdot 10^{16}$ & $2.8 \cdot 10^{14}$ & $3.0 \cdot 10^{16}$ \\
\hline
\end{tabular}


is possible with and without mutual neutralization, for the lower LOS (XL1) mutual neutralization is necessary for obtaining a good agreement. The enhancement caused by mutual neutralization is particulary pronounced for $6^{2} \mathrm{P}$ (indicated in figure 12 by vertical arrows): compared to the measurements the population densities from the CR model without mutual neutralization are too small by a factor of $3-3.5$. The effect of mutual neutralization is weaker for $7^{2} \mathrm{D}$ and negligible for $7^{2} \mathrm{P}$. This sequence fits very well to the results of the basic investigations presented in section 2.3 (shown in figure $10)$.

The electron temperatures used as input for the calculations are well within the error bar of the Langmuir probe results. The electron densities from the probe measurements could be used directly as model input. This very good agreement is somewhat surprising since - as described above - the position of the probes is different from the LOS used for OES. Additionally the probes determine the local plasma parameters close to the side walls while the OES results are averaged over the LOS.

The caesium ground state densities determined by the CR model have the same order of magnitude as the results of laser absorption spectroscopy. This agreement is quite good taking into account the different geometries of the LOS used for OES and laser absorption spectroscopy, discussed in section 3.2. The caesium ground state density along the upper LOS from the model is by a factor of 6.2 (without bias) or 18.7 (with bias) larger compared to the lower LOS. This upper/lower asymmetry of $n\left(6^{2} \mathrm{~S}\right)$ is higher than the typical asymmetry factors observed previously by laser absorption but smaller compared to the maximum measured values.

The negative hydrogen ion densities from the CR model $\left(10^{16} \mathrm{~m}^{-3}\right.$ for the upper LOS and $3 \cdot 10^{16} \mathrm{~m}^{-3}$ for the lower LOS) are in general agreement with the results of the laser detachment and cavity ring-down measurements based on different LOS. The value $10^{16} \mathrm{~m}^{-3}$ for $n\left(\mathrm{H}^{-}\right)$along the upper LOS represents an upper limit since - as can be seen in figure 12 - the influence of mutual neutralization on the population densities is almost negligible.

The much stronger influence of mutual neutralization on the population densities along the lower LOS (XL1) is caused by an asymmetry in the plasma parameters: direct excitation from the ground state $n\left(6^{2} \mathrm{~S}\right)$ is the dominant excitation channel for the upper LOS (XR1). Along the lower LOS (XL1) $n_{\mathrm{e}}$ is smaller and $n\left(\mathrm{H}^{-}\right)$is higher, resulting in a non-negligible relevance of the mutual neutralization channel.

Concluding, it is possible for all performed measurements to match the 
caesium population densities calculated by the CR model to the OES results. If the mutual neutralization process is taken into account the input parameters used by the $\mathrm{CR}$ model agree well with measurements performed in parallel with the OES measurements or during previous experimental campaigns. The CR model for caesium in low-temperature, low-pressure hydrogen-caesium plasmas of surface production based negative hydrogen ion sources can be used in a next step - for example - to determine the density of the singly charged positive caesium ion from measured line intensities (for known negative hydrogen ion density). Due to the high ionization degree of caesium precise knowledge of $n\left(\mathrm{Cs}^{+}\right)$is an important prerequisite for determining total caesium densities.

As shown in section 2.3 the increased population densities of the final states of the mutual neutralization process are distributed amongst the other excited states by means of interconnecting reactions. Thus, only CR models are suited to predict for all excited states the equilibrium resulting from these re-distribution processes. Corona models (taking into account the direct excitation channel only) are not sufficient to describe the population densities of the excited states of the caesium atom. However, a corona model extended by the mutual neutralization process can be applied to determine the population density of a single excited state if the influence of re-distributing the population densities of the excited states is small.

\section{Summary}

Based on the flexible solver Yacora a CR model for the caesium atom was constructed. This model focusses on the low-temperature, low-pressure hydrogen-caesium plasmas of surface production based negative hydrogen ion sources. The used set of input cross sections and transition probabilities is based on the most recent data available in the literature and oncorrecting and extrapolating these data where necessary. Cross sections for electron collision ionization and three-body recombination have been newly calculated based on the Gryzinski method and the Saha equation.

The most interesting reaction included in the model is the mutual neutralization of positive caesium ions and negative hydrogen ions: depending on the densities of the two involved particle species this process can significantly enhance the population of several excited states in the caesium atom and thus also the intensity of the emission lines originating from these excited 
states. In order to test this prediction, OES measurements were performed at the IPP prototype source for negative hydrogen ions.

A very good agreement of the CR model results with the population densities determined by OES could be obtained for two different LOS and in two different plasma regimes. The plasma parameters used as input for the CR model agree well with results from other diagnostic techniques.

These results verify the predicted high relevance of mutual neutralization and point out that population models for caesium in the electronegative plasma of the boundary layer in negative ion sources based on surface conversion (i.e. in plasmas with a high density of $\mathrm{H}^{-}$) need to take into account the mutual neutralization process. Depending on the field of application such models can either be an extended corona model or the present CR model. Since the latter takes into account several excited states and additionally the caesium ion, it represents an important prerequisite for deducing the total caesium density (including the population densities of the excited states and the density of the ion). Thus, the CR model might be - for example extremely useful for supporting the caesium conditioning process in surface production based negative hydrogen ion sources with the aim to obtain high and stable extracted values of the extracted negative ion current.

\section{Acknowledgments}

This project has received funding from the Euratom research and training programme 2014-2018.

The work was supported by a grant from Fusion for Energy (F4E-2008GRT-007) under the responsibility of Antonio Masiello. The opinions expressed herein are those of the authors only and do not represent the Fusion for Energys official position.

The authors would like to thank Professor Belyaev for useful discussions on the cross section database for caesium and for providing the mutual neutralization cross sections.

The authors wish to thank Professor Fantz for many useful discussions on populating mechanisms in the boundary layer of the IPP prototype negative hydrogen source.

\section{References}

[1] Michaelson, H.B.. J Appl Phys 1977;48:4729. 
[2] Braddick, H.J.J.. Rep Prog Phys 1960;23:154.

[3] Dimmock, J.O.. J Electron Mater 1972;1:255.

[4] Middleton, R.. Nucl Instr Methods 1974;122:35.

[5] Dudnikov, V.G.. Rev Sci Instrum 1992;63:2660.

[6] Moehs, D.P., Peters, J., Sherman, J.. IEEE T Plasma Sci 2005;33:1786.

[7] Kronberger, M., Küchler, D., Lettry, J., Midttun, Ø., ONeil, M., Paoluzzi, M., et al. Rev Sci Instrum 2010;81:02A708.

[8] Takeiri, Y., Kaneko, O., Tsumori, K., Oka, Y., Ikeda, K., Osakabe, M., et al. Nucl Fusion 2006;46:S199.

[9] Ikeda, Y., Umeda, N., Akino, N., Ebisawa, N., Grisham, L., Hanada, M., et al. Nucl Fusion 2006;46:S211.

[10] Masiello, A., Agarici, G., Bonicelli, T., Simon, M., Antoni, V., Esch, H.D., et al. Fusion Eng Des 2009;84:1276.

[11] Marcuzzi, D., Palma, M.D., M.Pavei, , Heinemann, B., Kraus, W., Riedl, R.. Fusion Eng Des 2009;84:1253.

[12] Fantz, U., Franzen, P., Kraus, W., Falter, H.D., Berger, M., ChristKoch, S., et al. Rev Sci Instrum 2008;79:02A511.

[13] Rasser, B., van Wunnik, J.N.M., Los, J.. Surf Sci 1982;118:697.

[14] Swanson, L.W., Strayer, R.W.. J Chem Phys 1968;48:2421.

[15] Ebbinghaus, G., Simon, A.. Chem Phys 1979;43:117.

[16] Gutser, R., Wimmer, C., Fantz, U.. Rev Sci Instr 2011;82:023506.

[17] Fröschle, M., Riedl, R., Falter, H., Gutser, R., Fantz, U., the NNBITeam, . Fusion Eng Des 2009;84:788.

[18] Fantz, U., Friedl, R., Fröschle, M.. Rev Sci Instrum 2012;83:123305.

[19] Gutser, R., Wünderlich, D., Fantz, U., the NNBI-Team, . Plasma Phys Control Fusion 2011;53:105014. 
[20] Fantz, U., Gutser, R., Wimmer, C.. Rev Sci Instrum 2010;81:02B102.

[21] Park, R.L., Lagally, M.G.. Methods of Experimental Physics, Solid State Physics: Surfaces. New York: Academic; 1985.

[22] Fantz, U., Falter, H., Franzen, P., Wünderlich, D., Berger, M., Lorenz, A., et al. Nucl Fusion 2006;46:S297.

[23] Norcross, D.W., Stone, P.M.. J Quant Spectrosc Radiat Transfer 1968;8:655.

[24] Sayer, B., Jeannet, J.C., Lozingot, J., Berlande, J.. Phys Rev A 1973;8:3012.

[25] Vogel, J.S.. AIP Conf Proc 2013;1515:89.

[26] Janev, R.K., Radulović, Z.M.. Phys Rev A 1978;17:889.

[27] Wünderlich, D., Dietrich, S., Fantz, U.. J Quant Spectrosc Radiat Transfer 2009;110:62.

[28] Speth, E., Falter, H.D., Franzen, P., Fantz, U., Bandyopadhyay, M., Christ, S., et al. Nucl Fusion 2006;46:S220.

[29] Bates, D.R., Kingston, A.E., McWirter, R.W.P.. Proc R Soc Lond A 1962;267:297.

[30] Fujimoto, T.. J Quant Spectrosc Radiat Transfer 1979;21:439.

[31] McWirter, R.W.P., Summers, H.P.. Applied Atomic Collision Physics. New York: Academic; 1984.

[32] Sansonetti, J.E.. J Phys Chem Ref Data 2009;38:761.

[33] Fantz, U., Falter, H., Franzen, P., Bandyopadhyay, M., Heinemann, B., Kraus, W., et al. Fusion Eng Des 2005;74:299.

[34] Behringer, K., Fantz, U.. New J Phys 2000;2:23.

[35] Sobel'man, I.I., Vainshtein, L.A., Yukov, E.A.. Excitation of Atoms and Broadening of Spectral Lines. Berlin: Springer; 1995.

[36] Krishnan, U., Stumpf, B.. At Data Nucl Data Tables 1992;51:151. 
[37] Chen, S.T., Gallagher, A.C.. Phys Rev A 1978;17:551.

[38] Gryzinski, M.. Phys Rev 1965;138:A336.

[39] Warner, B.. Mon Not R Astr Soc 1968;139:115.

[40] Norcross, D.W., Stone, P.M.. J Quant Spectrosc Radiat Transfer $1966 ; 6: 277$.

[41] Belyaev, A.K.. private communication; 2013.

[42] Mahmoud, M.A., Gamal, Y.E.E.. Optica Applicata 2010;40:129.

[43] Olsen, R.E., Kimura, M., Sato, H.. Phys Rev A 1984;30:1692.

[44] Kimura, M., Olsen, R.E., Pascale, J.. Phys Rev A 1984;30:1692.

[45] Greenland, P.T.. Proc Roy Soc Lond A 2001;457:1821.

[46] Hansen, L.K.. J Appl Phys 1964;35:254.

[47] Witting, H.L., Gyftopoulos, E.P.. J Appl Phys 1965;36:1328.

[48] Vainshtein, L., Opykhtin, V., Presnyakovn, L.. Sov Phys-JETP $1965 ; 20: 1542$.

[49] Ganas, P.S.. J Chem Phys 1982;76:2103.

[50] Zeman, V., McEachran, R.P., Stauffer, A.D.. J Phys B 1994;27:3175.

[51] Bartschat, K., Bray, I.. Phys Rev A 1996;54:1723.

[52] Kim, Y.K.. Phys Rev A 2001;64:032713.

[53] Nolan, J.F., Phelps, A.V.. Phys Rev 1965;140:A792.

[54] Zapesochni, I.P., Posti, E.N., Aleksakhin, I.S.. Sov Phys-JETP 1975;41:865.

[55] Surdutovich, E., Kauppila, W.E., Kwan, C.K., Miller, E.G., Parikh, S.P., Price, K.A., et al. Phys Rev A 2007;75:032720.

[56] McFarland, R.H.. Phys Rev 1965;139:A40. 
[57] Nygaard, K.J.. J Chem Phys 1968;49:1995.

[58] McGuire, E.J.. J Phys B 1997;30:1563.

[59] Łukomski, M., Sutton, S., Kedzierski, W., Reddish, T.J., Bartschat, K., Bartlett, P.L., et al. Phys Rev A 2006;74:032708.

[60] Janev, R.K., Reiter, D., Samm, U.. Collision Processes in LowTemperature Hydrogen Plasmas. Tech. Rep. JÜL-4105; Forschungszentrum Jülich, Jülich; 2003.

[61] Wünderlich, D.. Chem Phys 2011;390:75.

[62] Belyaev, A.K., Lebedev, O.V.. Phys Rev A 2011;84:014701.

[63] Christ-Koch, S., Fantz, U., Berger, M., the NNBI-Team, . Plasma Sources Sci Technol 2009;18:025003.

[64] Berger, M., Fantz, U., Christ-Koch, S., the NNBI-Team, . Plasma Sources Sci Technol 2009;18:025004.

[65] Wimmer, C., Fantz, U., the NNBI-Team, . AIP Conf Proc $2013 ; 1515: 246$.

[66] Hemsworth, R., Tanga, A., Antoni, V.. Rev Sci Instrum 2008;79:02C109.

[67] Franzen, P., Schiesko, L., Fröschle, M., Wünderlich, D., Fantz, U., the NNBI-Team, . Plasma Phys Control Fusion 2011;53:115006.

[68] Fantz, U., Franzen, P., Kraus, W., Berger, M., Christ-Koch, S., Falter, H., et al. Nucl Fusion 2009;49:125007.

[69] Wünderlich, D., Mochalskyy, S., Fantz, U., Franzen, P., the NNBITeam, . Plasma Sources Sci Technol 2014;23:015008.

[70] Schiesko, L.. Plasma Phys Control Fusion 2012;54:105002.

[71] Fantz, U., Wimmer, C.. J Phys D 2011;44:335202. 